\title{
Just the facts: Management of life-threatening bleeding with oral anticoagulant use in the emergency department
}

\author{
Michael L. Ho, MD*; Marc Carrier, MD MSc ${ }^{\dagger \ddagger}$
}

\section{CLINICAL SCENARIO}

A 63-year-old female is brought to the emergency department (ED) by ambulance after her partner noted progressive headache, vomiting, and gait instability starting six hours ago. Her past medical history includes atrial fibrillation and hypertension. Her medications include a "blood thinner," the name of which neither she nor her partner can recall. She is afebrile, with a blood pressure of $165 / 95$ and otherwise unremarkable vital signs. On examination, she is alert, oriented, and cooperative. Dysarthria is accompanied by a right-sided facial droop, and right-sided upper and lower extremity weakness is noted. A computed tomography (CT) scan of the brain reveals a $2 \times 2 \times 3 \mathrm{~cm}$ intracerebral hemorrhage in the left basal ganglia.

\section{KEY CLINICAL QUESTIONS}

\section{Should anticoagulation be reversed? What factors should be considered?}

Before reversal, it is important first to decide if the bleeding constitutes life-threatening or major bleeding as reversal strategies can cause adverse events such as thromboembolic complications. For example, most trials have used the International Society of Thrombosis and Haemostasis (ISTH) definition of major bleeding, which includes ${ }^{1}: 1$ ) fatal bleeding; 2) symptomatic bleeding in a critical area or organ such as intracranial, intraspinal, intraocular, retroperitoneal, intraarticular or pericardial, or intramuscular with compartment syndrome; and/or 3) bleeding causing a fall in hemoglobin level of $20 \mathrm{~g} / \mathrm{L}$ or more or leading to the transfusion of two or more units of whole blood or red cells.

In the highlighted case, this patient has intracranial bleeding and is, therefore, considered life-threatening.

Basic principles of management should include local and surgical (including embolization) hemostatic measures as appropriate while holding all antiplatelet and anticoagulants. Intravenous (IV) tranexamic acid can also be considered. In cases of acute overdose, activated charcoal can be also be considered, particularly if within one or two hours of ingestion.

For warfarin, it is important to determine the international normalized ratio (INR) level, which would help guide reversal agent dosing (Table 1). For direct oral anticoagulants (DOACs), which include dabigatran, rivaroxaban, apixaban, and edoxaban, it is important to determine renal function and time since the last dose of the anticoagulant. With normal renal function, the half-life of dabigatran, rivaroxaban, apixaban, and edoxaban are approximately 14,8 , 12 , and 10 hours, respectively. ${ }^{2}$ After three to five half-lives, the anticoagulant effects are minimal. The real-time anticoagulant effect can be measured with DOACs but are not as straightforward as INR with warfarin and will be discussed below.

From *Department of Emergency Medicine, University of Ottawa, Ottawa, Ontario; †Department of Medicine, Division of Hematology, University of Ottawa, Ottawa, Ontario; and ¥The Ottawa Hospital Research Institute, University of Ottawa, Ottawa, Ontario.

Correspondence to: Dr. Michael L. Ho, 1053 Carling Ave., E-Main, Room EM-206, Box 227, Ottawa, Ontario, K1Y 4E9; Email: miho @toh.ca 


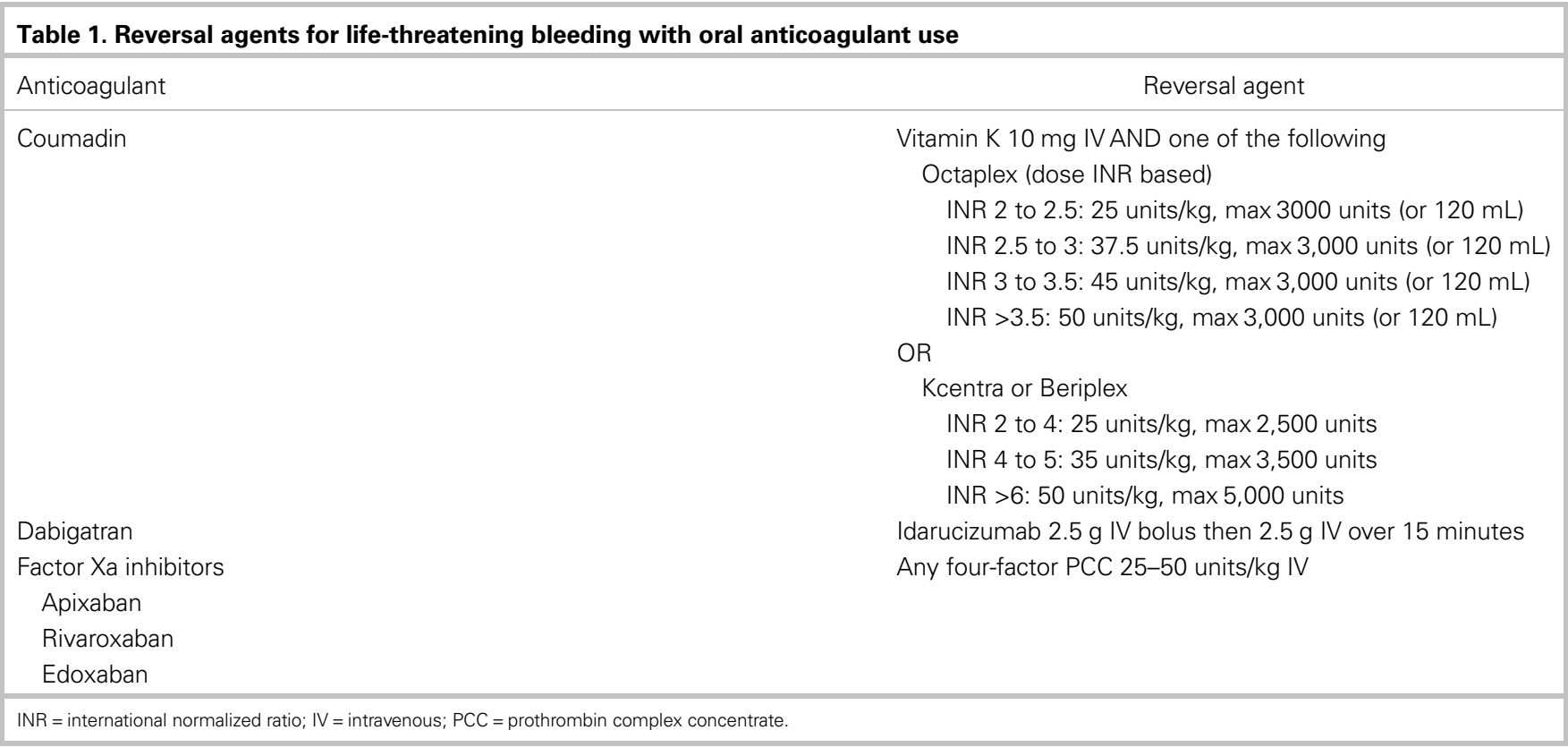

\section{If this patient's "blood thinner" is warfarin, how should the anticoagulant effects be reversed?}

Warfarin is a vitamin $\mathrm{K}$ antagonist that depletes levels of factors II, VII, IX, X, and protein C and S, resulting in less downstream thrombin, fibrin, and cross-linked fibrin clots. Its anticoagulant activity is represented in the INR.

Reversal of warfarin in life-threatening bleeding is typically performed with vitamin $\mathrm{K}, 10 \mathrm{mg} \mathrm{IV}$, administered over 20-30 minutes and IV prothrombin complex concentrate (PCC). In Canada, PCC is a four-factor variant that contains factors II, VII, IX, X, and protein C and S, as well as a small amount of heparin. The dose of PCC is dependent on both the INR and the specific product being used (see Table 1). Typical doses for PCC are 25-50 units $/ \mathrm{kg}$, to a maximum of 3000 units.

Thromboembolic complications can occur with PCC in approximately $1.8 \%$ of patients. ${ }^{3}$ PCC also contains trace doses of heparin and should not be given to patients with heparin-induced thrombocytopenia (HIT). PCC is a blood product, and the usual considerations apply.

If PCC is not available, fresh frozen plasma can be used in lieu of PCC. However, reversal doses of plasma are typically in the $4-6$ unit range (1-1.5 L of plasma) and require time to thaw.

\section{If this patient's "blood thinner" is dabigatran, how should the anticoagulant effects be reversed?}

Dabigatran is a direct thrombin (factor IIa) inhibitor. Its anticoagulant effect is not easily measured with commonly available lab tests. However, a normal activated partial thromboplastin time (aPTT) probably excludes excess levels of dabigatran, whereas a normal thrombin time (TT) or dilute thrombin time (dTT) excludes clinically relevant dabigatran anticoagulant activity. ${ }^{2}$

Reversal of dabigatran's anticoagulant effect is performed using idarucizumab, which is a monoclonal antibody that specifically binds dabigatran and thrombin-bound dabigatran. Idarucizumab is administered as a $2.5 \mathrm{~g}$ IV bolus, followed by another $2.5 \mathrm{~g}$ administered over 15 minutes. ${ }^{4}$ Duration is approximately 24 hours, and further dosing could be warranted depending on the clinical context.

The safety of idarucizumab has not been robustly studied; however, trials to date suggest thromboembolic complications in the range of $5 \%$. 


\section{If this patient's "blood thinner" is apixaban, rivaroxaban, or edoxaban, how should the anticoagulant effects be reversed?}

Apixaban, rivaroxaban, and edoxaban are oral factor Xa inhibitors, and the differences between them are minimal. At present, it is not usually easy to measure their level of anticoagulant effect. Some institutions have specific anti-Xa assays that are calibrated to each of these drugs, thereby indicating anticoagulant activity.

In Canada, there is no specific reversal agent for oral factor Xa inhibitors. Currently, the most accepted strategy to treat life-threatening bleeding with these agents is to administer high doses of intravenous PCC ( 25 to $50 \mathrm{units} / \mathrm{kg}$ ) to "overload" the coagulation cascade to achieve hemostasis. ${ }^{5}$

In the United States, the Food and Drug Administration (FDA) approved a drug named andexanet alfa, which is a modified form of factor Xa that acts as a "decoy" receptor and binds and deactivates the oral factor Xa inhibitors.

\section{Are there any online tools that can help guide clinical decision making in cases of bleeding with oral anticoagulant use?}

The Thrombosis Canada Bleed Management tool estimates anticoagulant levels and guides management in individual cases by combining patient and laboratory parameters. ${ }^{6}$ The tool is free to use and available at https://thrombosiscanada.ca/tools/?calc=vivomap271.

\section{CASE RESOLUTION}

The patient's intracranial bleeding is life-threatening and supportive care measures including raising the head of the bed to 30 degrees are initiated. You decide that her anticoagulation should be reversed despite risks of thromboembolic complications. You confirm that her anticoagulant is dabigatran and that her last dose was six hours ago. To reverse the effects of dabigatran, you administer idarucizumab $2.5 \mathrm{~g}$ IV as a bolus and another $2.5 \mathrm{~g}$ IV over 15 minutes. As an adjunct, you also administer tranexamic acid $1 \mathrm{~g}$ IV over 20 minutes. Additionally, you requested an urgent consultation with a neurosurgeon.

\section{KEY POINTS}

- Life-threatening bleeding associated with oral anticoagulant use should first be managed with supportive care and local hemostatic measures.

- With warfarin, it is important to measure an INR to help guide dosing of a reversal agent. Reversal is performed using vitamin $\mathrm{K}, 10 \mathrm{mg} I \mathrm{~V}$, and PCC at 25-50 units $/ \mathrm{kg} \mathrm{IV}$.

- It is important to determine renal function and time since the last dose for dabigatran, apixaban, rivaroxaban, and edoxaban. With normal renal function, their half-lives are 14, 8, 12, and 10 hours, respectively. Anticoagulant effects are minimal after 3-5 half-lives.

- With dabigatran, a normal aPTT probably excludes excess levels of dabigatran, but a TT or dTT excludes clinically relevant dabigatran anticoagulant activity. Reversal is performed using idarucizumab $2.5 \mathrm{~g}$ IV as a bolus followed by $2.5 \mathrm{~g}$ IV over 15 minutes.

- With apixaban, rivaroxaban, or edoxaban, the anticoagulant activity can be measured using specifically calibrated anti-Xa assays, though these assays are not commonly available. Reversal is performed using PCC at $25-50$ units $/ \mathrm{kg}$. 
- The main complication when reversing the effects of oral anticoagulants is thromboembolic complications, which can occur approximately in $2 \%-5 \%$ of cases. Aggressive reversal strategies should, therefore, be reserved for life-threatening bleeding.

- The Thrombosis Canada Bleed Management tool is a free online tool that estimates anticoagulant levels and guides clinical management in cases of bleeding with oral anticoagulant use.

Keywords: Bleeding, emergency department, oral anticoagulant

Competing interests: None.

\section{REFERENCES}

1. Schulman S, Angerås U, Bergqvist D, et al. Definition of major bleeding in clinical investigations of antihemostatic medicinal products in surgical patients. 7 Thromb Haemost 2010;8(1):202-4.

2. Husted S, Verheugt FW, Comuth WJ. Reversal Strategies for NOACs: State of Development, Possible Clinical Applications and Future Perspectives. Drug Saf 2016;39(1):5-13.

3. Dentali F, Marchesi C, Giorgi Pierfranceschi M, al. Safety of prothrombin complex concentrates for rapid anticoagulation reversal of vitamin K antagonists. A meta-analysis. Thromb Haemost 2011;106(3):429-38.

4. Pollack Jr CV, Reilly PA, van Ryn J, et al. Idarucizumab for dabigatran reversal - full cohort analysis. NEngl7 Med 2017;377(5):431-41.

5. Schulman S, Gross PL, Ritchie B, et al. Prothrombin complex concentrate for major bleeding on factor Xa inhibitors: a prospective cohort study. Thromb Haemost 2018;118(5):842-51.

6. Thrombosis Canada. Bleed Management Tool. https://thrombosiscanada.ca/tools/?calc=vivomap271 (accessed February 25, 2019). 